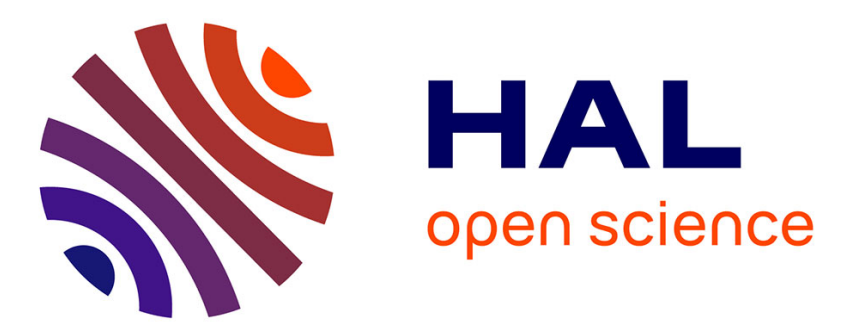

\title{
Un environnement personnel d'apprentissage évaluant des distances épistémiques et dialogiques
}

\author{
Philippe Dessus, Ștefan Trăușan-Matu, Fridolin Wild, Damien Dupré, \\ Mathieu Loiseau, Traian Rebedea, Virginie Zampa
}

\section{- To cite this version:}

Philippe Dessus, Ștefan Trăușan-Matu, Fridolin Wild, Damien Dupré, Mathieu Loiseau, et al.. Un environnement personnel d'apprentissage évaluant des distances épistémiques et dialogiques. Distances et savoirs, 2011, 9 (4), pp.473-492. 10.3166/ds.9.473-492 . hal-01079177

\section{HAL Id: hal-01079177 \\ https://hal.science/hal-01079177}

Submitted on 18 Jun 2018

HAL is a multi-disciplinary open access archive for the deposit and dissemination of scientific research documents, whether they are published or not. The documents may come from teaching and research institutions in France or abroad, or from public or private research centers.
L'archive ouverte pluridisciplinaire HAL, est destinée au dépôt et à la diffusion de documents scientifiques de niveau recherche, publiés ou non, émanant des établissements d'enseignement et de recherche français ou étrangers, des laboratoires publics ou privés. 


\title{
Un environnement personnel d'apprentissage évaluant des distances épistémiques et dialogiques
}

\author{
Philipe Dessus $^{1}$ - Stefan Trausan-Matu ${ }^{2}$ - Fridolin Wild ${ }^{3}$ \\ Damien Dupré $^{4}$ - Mathieu Loiseau ${ }^{1,5}$ - Traian Rebedea ${ }^{2}$ \\ Virginie Zampa ${ }^{5}$
}

1. LSE \& IUFM-UJF, 1251 av. Centrale, BP 47, 38040 Grenoble cedex 9, France philippe.dessus@upmf-grenoble.fr

2. Université Politehnique, 313 Splaiul Indendentei, Bucarest, Roumanie \{stefan.trausan,traian.rebedea\}@cs.pub.ro

3. Knowledge Media Institute, The Open University, Walton Hall MK76AA Milton Keynes, UK-f.wild@open.ac.uk

4. Laboratoire interuniversitaire de psychologie, 1251 av. Centrale, BP 47 38040 Grenoble, France cedex 9 -damien.dupre@ixiade.com

5. LIDILEM, Université Stendhal, BP 25, 38040 Grenoble cedex 9, France \{mathieu.loiseau,virginie.zampa\}@u-grenoble3.fr

RÉSUMÉ. Tout enseignement est à distance. De nouveaux environnements informatisés peuvent évaluer automatiquement diverses productions d'apprenants et leur procurer divers renseignements sur ces dernières. En révélant d'autres distances que physiques (épistémiques et sociales), et en s'agrégeant au sein de plates-formes sociales, ces environnements offrent de nouvelles perspectives dans l'enseignement à distance et en présence. Cet article présente trois widgets évaluant des aspects divers des processus d'apprentissage et délivrant leurs évaluations sous la forme de distances (distance sémantique, cognitive individuelle ou sociale). Ces trois widgets peuvent s'interconnecter pour former un environnement personnel d'apprentissage, environnement qui a fait l'objet d'une première étude exploratoire d'usage, avec des étudiants d'informatique.

ABSTRACT. Every teaching is distant. New computer environments can automatically assess various productions of learners and provide several feedback. By revealing non-physical distances (social and epistemic), and by joining in social platforms, these environments offer new opportunities in distance learning but also in face-to-face learning. This article presents three widgets evaluating various aspects of learning processes and delivering their evaluation as a form of distance (semantic distance, individual cognitive distance and social distance). All three widgets can be interconnected to build a personal learning environment, which is the subject of a first exploratory case-study with computer science students.

D\&S - 2011 - Où va la distance ? 
MOTS-CLÉS: environnements d'apprentissage personnels, rétroactions, distance épistémique, distance sociale, analyse sémantique latente.

KEYWORDS: personal learning environments, feedback, epistemic distance, social distance, latent semantic analysis.

DOI:10.3166/DS.9.473-492 @ Cned/Lavoisier 2011

\section{Introduction}

Toute situation d'enseignement est à distance ; même celles communément dites «en présence» le sont, ne serait-ce que du fait de l'existence d'une distance épistémique nécessaire entre ce que sait l'enseignant et ce que sait l'élève (Dessus, 2008). Ce qui caractérise généralement les situations d'enseignement à distance est un éloignement physique et temporel des participants (enseignants, tuteurs, apprenants) qui rend leurs interactions différentes de celles en présence. De nombreux modèles théoriques (p. ex., la distance de transaction de Moore, 1993), mais aussi de nombreux outils et systèmes ont été conçus pour analyser, modéliser, et parfois agir sur cette distance, afin de rendre la tâche de chaque participant plus aisée et parfois plus proche de ce qu'elle serait dans une situation en présence. Ces modèles, ainsi que des récents travaux en psychologie ont montré que les différentes dimensions de la distance (temporelle, spatiale, mais aussi sociale ou cognitive) étaient inter-reliées (Trope et Liberman, 2010).

Les différentes théories sur la distance, dans le domaine de l'enseignement $\mathrm{du}$ même nom, partagent deux inconvénients : elles sont principalement centrées sur les aspects de communication, et ne s'intéressent pas particulièrement à la connaissance enseignée. De plus, elles ne comportent pas toujours de critères mesurables, si on se réfère aux rares tentatives de rendre quantifiable la distance de transaction (Dessus, 2003 ; Saba et Shearer, 1994). Nous pensons toutefois que, dans les processus d'enseignement/apprentissage à distance, il existe des distances épistémiques et cognitives qui peuvent jouer des rôles importants. Il est également possible de rendre compte de ces distances, non en mètres ou avec des chronomètres, mais avec des outils de traitement automatique de la langue (p. ex., Dessus et al., 2009 ; Trausan-Matu et Dessus, 2010). Ainsi, la récente intégration de ces outils dans les plates-formes d'enseignement à distance pourrait apporter de nouvelles réponses à la question qui fédère ce numéro spécial, «Où va la distance ? »: Quels nouveaux types de distances peuvent-ils mesurer? Pour promouvoir quels types d'activités d'apprentissage et d'enseignement?

Dans les contextes typiques d'enseignement à distance, les étudiants réalisent de très nombreuses activités : entre autres, ils ont à lire des documents de cours, à en produire des résumés ou des synthèses afin de les apprendre, à produire des blogs réflexifs les amenant à prendre de la distance (sic) avec les contenus enseignés, à discuter de ces contenus entre pairs et/ou avec l'enseignant. Pour chacune de ces activités les étudiants ont besoin de retours évaluatifs leur permettant d'améliorer 
leurs productions et/ou leurs connaissances. Les outils actuellement disponibles sur le web, comme le courriel ou la messagerie instantanée (chat) permettent et encouragent l'enseignement collaboratif assisté par l'ordinateur (Stahl, 2006), et donc la diffusion de rétroactions humaines. L'enseignement à distance ne réduit pas seulement les distances physiques via l'utilisation des outils susnommés. Il est essentiellement fondé sur un paradigme spécifique : le dialogisme, la participation polyphonique, en lequel plusieurs «voix» réduisent les distances entre les collaborateurs, et entrent en inter-animation (Trausan-Matu et al., 2007). Des environnements informatiques peuvent procurer aux apprenants des rétroactions automatiques sur une partie de leur production sans aucune intervention d'un enseignant ou d'un tuteur, ce qui permet à l'apprenant d'avoir des rétroactions plus régulières et instantanées, et à l'enseignant de se focaliser sur des problèmes spécifiques (p. ex., à propos du cours, des apprenants).

Quels nouveaux types de «distances» peuvent être révélés dans des environnements informatiques? Quels sont les types d'apprentissages qui pourront être réalisés en combinant ces derniers ? Ces environnements, très complexes et dont le comportement est encore peu prédictible par les apprenants, sont-ils vraiment utilisables? Le but de cet article est d'esquisser une réponse à ces questions. Il s'agit dans un premier temps de définir de manière simple ce qu'est un environnement personnel d'apprentissage et les dimensions selon lesquelles il pourrait favoriser les activités d'apprentissage ou d'enseignement. Puis, nous présentons trois outils logiciels, conçus pour être autant de widgets (window gadgets, petites applications spécialisées dans une tâche et pouvant interagir les unes avec les autres) en interaction dans une plate-forme sociale d'enseignement (p. ex., Elgg), jouant le rôle d'un environnement personnel d'apprentissage. Nous terminerons par la description d'une étude liminaire de l'utilisation de ces outils en contexte d'apprentissage collaboratif.

\section{Apprendre à distance dans un environnement informatisé}

Un environnement (qu'il soit informatisé ou non) peut permettre l'apprentissage s'il satisfait aux conditions suivantes (reprises de Dessus, 2008) :

- l'environnement doit présenter une situation de travail spécifiquement aménagée qui donne de bonnes conditions à l'apprenant: ce dernier effectue, de manière plus ou moins guidée, des «déplacements» (moves) dans cet environnement qui sont scrutés et interprétés par l'enseignant et ses pairs ;

- dans cette situation, l'apprenant doit avoir accès à des connaissances (p. ex., par le biais de l'enseignant ou de textes présentés d'une quelconque manière, par livre ou ordinateur) ;

- il faut que les tâches que l'apprenant réalise dans cette situation soient soigneusement sélectionnées par l'enseignant : elles doivent être variées, répétables, ni trop difficiles ni trop simples au regard du niveau de l'apprenant, et bien sûr ne pas présenter un quelconque risque ou danger ; 
- l'apprenant doit avoir une certaine activité de communication avec l'enseignant (ou d'interaction avec le livre), de manière à ce que les connaissances puissent être communiquées. Cette activité recouvre des formes diverses (demande d'explications supplémentaires, discussion, restitution orale, production écrite, etc.) ;

- l'apprenant doit avoir un certain retour à propos de son activité dans la situation (ce qu'il fait, où il va, ce qu'il lui reste à apprendre), ce retour étant fondé sur le produit de l'activité de communication exposée ci-avant ;

- l'apprenant interagit avec ses pairs, dans des situations plus ou moins guidées par l'enseignant, de manière à négocier et construire un point de vue partagé (Stahl, 2006).

Il est intéressant de noter que, pour beaucoup des conditions ci-dessus, la notion de distance intervient, que ce soit la distance «parcours » dans l'environnement, celle entre tâches et connaissances antérieures de l'apprenant, celle entre activité prescrite et réelle, etc. Certaines de ces distances sont matérielles, d'autres sont épistémiques. Ce sont ces dernières qui nous intéressent plus précisément ici. Deux objets théoriques, lorsqu'ils sont implantés dans des systèmes, permettent d'exploiter cette distance : les boucles et écarts.

Une boucle, selon notre définition (Dessus, 2011), est une partie du flux de travail d'un apprenant dans lequel ce dernier a une idée du chemin à parcourir. Pour cela des indications explicites ou implicites lui sont fournies afin qu'il ait une idée de là où il est, des buts qui lui sont fixés, de ceux qu'il a l'intention d'atteindre, etc. L'écart représente le degré de différence entre les informations traitées. Si les deux informations sont identiques, alors l'apprenant peut considérer que la tâche objet du flux de travail est terminée, si elles sont différentes il peut continuer à travailler (Carver et Scheier, 2011). Comme l'indiquent ces derniers auteurs, l'écart entre le réalisé et l'intention ne décroit pas nécessairement avec le temps, il est donc nécessaire d'en procurer à l'apprenant une mesure précise. Certains systèmes peuvent informer leur utilisateur sur la manière dont il progresse (quels progrès fait-il par rapport à son but). Ces trois questionnements (« où vais-je ?», « comment y aller ? » et « que puisje faire ensuite?») sont au cœur de tout processus de rétroaction (Hattie et Timperley, 2007). Ainsi, en utilisant un système informatisé, tout apprenant est inséré dans un flux de travail composé de différentes boucles. Il est important, également, de noter que l'utilisateur est libre de quitter la boucle à tout moment

\section{Les environnements personnels d'apprentissage}

Un environnement personnel d'apprentissage (EPA, ou PLE pour Personal Learning Environment) peut être défini comme le «[...] réseau de personnes entourant un individu, ces personnes utilisant des artefacts et outils pendant qu'ils sont impliqués dans des activités isolées ou collaboratives de construction de connaissances et d'informations plus ou moins intentionnelles » (Wild et al., 2011, p. 39). L'EPA a tout d'abord été un concept technique dans lequel l'apprenant occupe une place centrale, auparavant occupée par le serveur dans les plates-formes 
d'apprentissage classiques (Olivier et Liber, 2001). Pendant la dernière décennie, la recherche sur les EPA s'est élargie en passant d'aspects strictement techniques à des considérations pédagogiques importantes, comme celle de favoriser l'apprentissage autorégulé (Attwell, 2007). D'un point de vue technique, les widgets (applications web miniatures et centrées sur une tâche) sont apparus et ont favorisé la combinaison et l'interopérabilité d'outils distribués dédiés à une activité d'apprentissage. On peut concevoir les widgets de façon à ce qu'ils communiquent entre eux et partagent des informations, permettant à leurs utilisateurs de réaliser une activité favorisée par cette utilisation combinée. Dans le cadre de la recherche présentée ici, une plateforme Elgg fait office d'EPA. Les utilisateurs peuvent y sélectionner des widgets généralistes (comme des gadgets Google), mais également des widgets conçus pour leur apprentissage. Ainsi, l'environnement de travail peut être agencé selon les besoins de chaque apprenant et peut s'interfacer avec toute autre application de l'EPA.

\section{Présentation des widgets et de leurs interconnexions au sein d'un Environnement personnel d'apprentissage}

Les outils (resp. Pensum, CONSPECT et PolyCAFe) présentés maintenant se centrent sur trois aspects épistémiques différents de la distance en en produisant des mesures :

- une distance sémantique impliquant les phrases du texte produit (intraproduction): Pensum détecte les sauts de cohérence (distance entre phrases successives du texte produit), les phrases hors-sujet et les phrases possiblement pertinentes (distance entre phrases du texte produit et des textes-sources) dans les productions des étudiants (essais, résumés, notes de cours) ;

- une distance cognitive individuelle entre productions : CONSPECT analyse les productions réflexives des étudiants, p. ex., $b \log s$, en affiche l'évolution dans le temps, ou encore en compare le contenu avec ceux d'autres blogs (expert, émergent, de référence, de pair). Il affiche également des informations sur les relations entre concepts et leur occurrence ;

- une distance cognitive sociale : PolyCAFe analyse le contenu des tours de parole dans les chats ou forums et représente l'apport de chaque protagoniste et le degré de collaboration, de polyphonie entre eux.

À leur façon, ces trois systèmes tentent donc de donner une nouvelle vision de ce que pourraient être des "distances » épistémiques et dialogiques, c'est-à-dire plus centrées sur la connaissance que sur la distance physique ou temporelle. Ces trois systèmes ont été conçus et implémentés dans le cadre du projet européen LTfLL (Language Technologies for Lifelong Learning, http://www.ltfll-project.org/). 


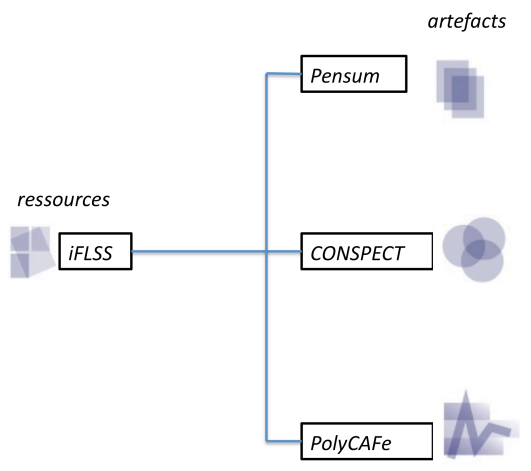

Figure 1. Le parcours possible d'un apprenant dans les différents widgets

\begin{tabular}{|c|c|c|c|}
\hline Widget & $\begin{array}{l}\text { Tâches préalables } \\
\text { requises }\end{array}$ & $\begin{array}{l}\text { Tâches des } \\
\text { apprenants }\end{array}$ & Types de rétroactions sur la distance \\
\hline Pensum & $\begin{array}{l}\text { Rechercher des } \\
\text { textes de cours et } \\
\text { les sélectionner }\end{array}$ & $\begin{array}{l}\text { Lire des } \\
\text { textes de } \\
\text { cours et en } \\
\text { réaliser des } \\
\text { synthèses }\end{array}$ & $\begin{array}{l}\text { Distances épistémiques intraproduction : } \\
\text { - Distance sémantique entre deux phrases } \\
\text { de la synthèse (cohérence) } \\
\text { - Distance sémantique entre chaque phrase } \\
\text { d'un cours donné et la synthèse } \\
\text { (pertinence) } \\
\text { - Distance entre chaque phrase de la } \\
\text { synthèse et le cours (détection de hors- } \\
\text { sujet) }\end{array}$ \\
\hline $\begin{array}{l}\text { CONS- } \\
\text { PECT }\end{array}$ & $\begin{array}{l}\text { Produire un texte } \\
\text { à propos de sa } \\
\text { compréhension d'un } \\
\text { cours (blog, essai, } \\
\text { résumé, synthèse) }\end{array}$ & $\begin{array}{l}\text { Comparer } \\
\text { les concepts }\end{array}$ & $\begin{array}{l}\text { Distance épistémiques interproductions : } \\
\text { - comparaison avec des productions } \\
\text { précédentes ou avec un pair ou un expert } \\
\text { - similitude des concepts mentionnés } \\
\text { - poids des concepts mentionnés }\end{array}$ \\
\hline $\begin{array}{l}\text { Poly- } \\
\text { CAFe }\end{array}$ & $\begin{array}{l}\text { Réaliser une } \\
\text { discussion } \\
\text { collaborative ou un } \\
\text { clavardage à propos } \\
\text { de notions du cours }\end{array}$ & $\begin{array}{l}\text { Réfléchir } \\
\text { sur la } \\
\text { discussion } \\
\text { et préparer } \\
\text { les discus- } \\
\text { sions futures }\end{array}$ & $\begin{array}{l}\text { Distance sociale : } \\
\text { - visualisation liens entre tours de parole } \\
\text { de participants à une discussion } \\
\text { - analyse des actes de parole de chaque } \\
\text { tour de parole } \\
\text { - analyse de la participation } \\
\text { - distance épistémique entre les } \\
\text { participants de la discussion }\end{array}$ \\
\hline
\end{tabular}

Tableau 1. Quelques caractéristiques des widgets présentés dans l'article

Comme déjà évoqué, la notion d'environnement personnel d'apprentissage implique une certaine modularité (installation d'outils) et une forme 
d'interopérabilité (navigation au sein des outils). Les outils que nous avons conçus sont aptes à communiquer entre eux et permettent à l'apprenant des usages et parcours variés. La figure 1 décrit un parcours possible dans les différents outils. L'apprenant commence par un accès à différentes ressources par iFLSS, un moteur de recherche fondé sur des ontologies (Westerhout et al., 2010) que nous ne décrirons pas ici. Ce moteur lui permet d'avoir accès à différents documents, présentations, vidéos, ressources bibliographiques liés à un contenu donné par motsclés, que ce soit dans les ressources web 2.0 disponibles (ressources YouTube, SlideShare ou Del.icio.us) ou même dans le réseau social créé par l'apprenant, et qui peut donc être composé de ses pairs. La lecture et le travail sur ces documents l'amène ensuite à travailler via les trois autres environnements.

Le tableau 1 reprend les principales caractéristiques de l'environnement d'apprentissage mis en place dans le cadre de notre projet et indique widget par widget, les tâches que les apprenants les utilisant peuvent remplir, ainsi que les types de rétroactions qu'ils délivrent. Passons maintenant à leur description individuelle.

\section{Pensum, un widget d'analyse de synthèses de cours}

Pensum (Loiseau et al., 2011) est un système d'aide à la rédaction de synthèses. Il vise à laisser l'apprenant relativement libre dans son apprentissage, tout en lui permettant, à tout moment, de prendre une part active à son évaluation (Butler et Winne, 1995). Pour ce faire, l'apprenant peut demander au système un retour sur son travail, qui lui fournira des rétroactions de trois types:

- Saut de cohérence : deux phrases consécutives de la synthèse ne semblent pas s'enchaîner correctement ('ไ' dans la figure 2);

- Idée non couverte : les phrases du cours qui ne semblent pas avoir été prises en compte dans la synthèse sont indiquées en gris clair (phrase commençant par «L'annuaire... »);

- Phrase non pertinente : les phrases de la synthèse qui semblent sans relation avec aucun élément des textes-sources sont indiquées en gris clair (phrases commençant par «Dans le premier document...» ou par «Dans ces conditions...»).

Chacune de ces rétroactions utilise LSA - Latent Semantic Analysis, une méthode de comparaison sémantique textuelle, voir Landauer et Dumais (1997). Les rétroactions ne pouvant être correctes à $100 \%$ et l'objectif du système étant de permettre à l'apprenant d'avoir un regard réflexif sur son propre travail, un ensemble de fonctionnalités a été développé pour permettre à l'apprenant de remettre en question ou contredire les jugements du système et dont les traces sont conservées tout au long de la tâche de rédaction de la synthèse. Pensum donne à l'apprenant un premier ensemble de distances, toutes épistémiques, lui permettant d'analyser sa compréhension du contenu du cours. La mention de sauts de 
cohérence, en soulignant un possible écart sémantique trop important entre deux phrases, amène l'apprenant à préciser sa pensée et réduire cet écart. La mention d'idées non couvertes (phrases du texte-source) l'amène à diagnostiquer l'importance de la phrase en question, donc à reconsidérer sa possible inclusion dans le résumé. Enfin, la mention de phrases non pertinentes (hors-sujet) peut être un moyen pour l'apprenant de repérer une compréhension erronée du cours.

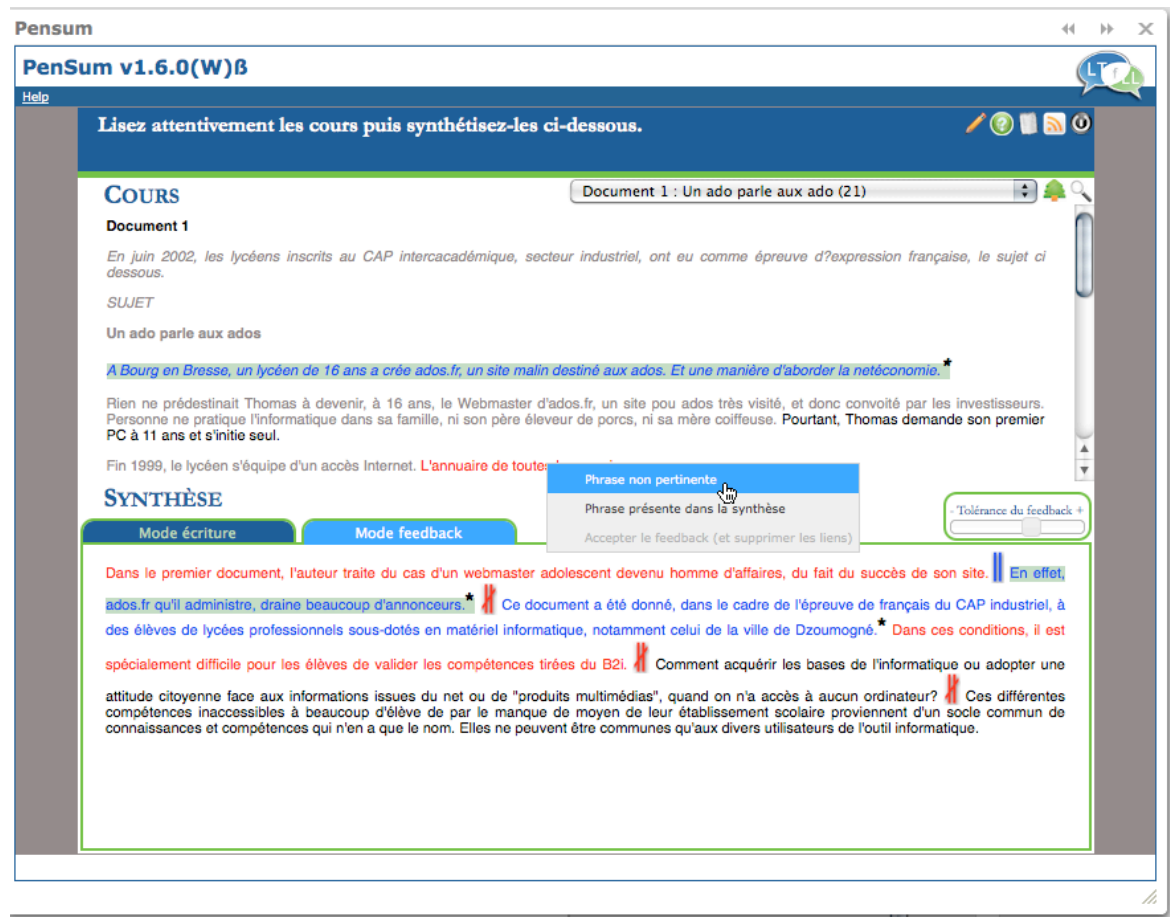

Figure 2. Interface de Pensum. La zone supérieure affiche le cours (document par document) et la zone inférieure accueille la saisie de la synthèse, en deux modes alternatifs : écriture ou feedback. Les phrases remises en question par l'apprenant sont en gris foncé (commençant par "Ce document... »), la justification de la remise en question par liaison explicite entre une phrase de la synthèse et du cours est suivie d'un astérisque et surlignée, les phrases non pertinentes pour la synthèse sont en gris dans la zone de texte "cours»

\section{CONSPECT, un widget analysant le développement conceptuel des apprenants}

CONSPECT (voir p. ex. Berlanga et al., à paraître) est un widget qui permet à un apprenant de superviser le développement de ses connaissances durant un cours ou un intervalle de temps plus long (année universitaire). CONSPECT via une analyse automatique également fondée sur LSA, met en évidence par un conceptogramme 
(voir figure 3) les liaisons existant entre les concepts utilisés par l'apprenant mais également les différences entre apprenants. L'utilisation de CONSPECT suppose qu'un texte ait été préalablement saisi, et que ce texte représente un état des connaissances de l'apprenant (sous la forme d'un blog, d'un essai, d'un résumé). Il est possible, bien que non obligatoire, que ce texte soit issu de Pensum. En faisant la comparaison entre deux éléments de production, CONSPECT affiche les possibles concepts apparaissant dans l'une des productions seulement (concepts manquants) ainsi que ceux apparaissant dans les deux (concepts similaires).

La figure 3 montre un tel exemple de comparaison entre pairs du « recoupement» conceptuel entre deux productions écrites d'étudiants. Chaque cercle représente un concept extrait des textes et les rectangles symbolisent les apprenants. Un concept est connecté à un apprenant s'il est présent dans l'un des billets de blog de ce dernier. Les concepts sont d'autant plus proches les uns des autres qu'ils sont associés dans les textes des apprenants. À partir de ces données, CONSPECT utilise un algorithme de dessin fondé sur les forces pour mettre en forme les conceptogrammes : les nœuds (utilisateurs, concepts) se repoussent et les liens entre les nœuds (concepts apparaissant dans les mêmes billets, utilisateurs ayant recours aux concepts) sont présentés comme des ressorts, l'algorithme utilise des lois de mécanique et positionne les éléments selon le bilan des forces. In fine, les étudiants et les concepts seront représentés dans la même zone selon l'utilisation des seconds par les premiers. Les concepts étant extraits par la méthode de l'analyse sémantique latente (LSA), ils sont relativement indépendants des mots y référant. Par exemple, un texte contenant le mot « automobile» peut être représenté par le concept étiqueté « voiture ».

Dans l'exemple de la figure 3, plusieurs étudiants ont blogué à propos d'un cas médical, un patient avec un problème d'alcool. La représentation des concepts dans l'espace montre que trois des étudiants (représentés par les trois carrés du haut de la figure) sont très proches. Une interaction avec le widget permet d'accéder à leurs textes et d'analyser leurs productions. La copie d'écran montre également que les trois autres étudiants sont atypiques, car ayant une représentation périphérique et faiblement connectée aux concepts. Une inspection de leur production a montré que deux d'entre eux n'ont pas fait une description du cas très précise et que la production du troisième était en fait le billet excessivement concis utilisé par le tuteur de ce groupe pour mettre en place l'activité.

Les distances analysées par CONSPECT sont différentes de celles de Pensum. Alors que ce dernier analyse des distances textuelles questionnant la compréhension de l'apprenant, CONSPECT représente visuellement trois nouvelles distances à propos de concepts mentionnés dans les productions des apprenants: une perspective d'évolution dans l'utilisation de tel ou tel concept permettant une comparaison (avant-après ou entre pairs) ; une perspective de similitude (proximité des concepts entre eux); et enfin une perspective de poids relatif (la taille des concepts représentés est fonction de leur occurrence). 


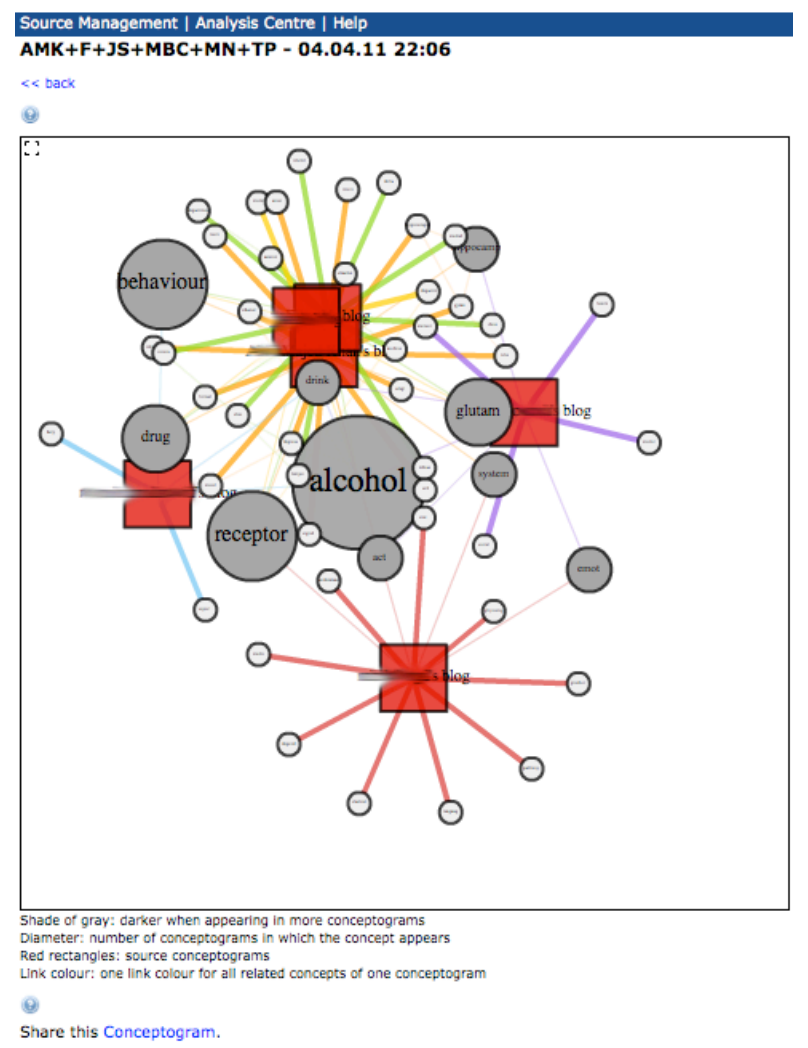

Figure 3. L'interface de CONSPECT. Les cercles représentent les différents concepts présents dans les productions des apprenants, les carrés représentent les apprenants

\section{Description de PolyCAFe, un widget d'analyse de discussions collaboratives}

L'utilisation de PolyCAFe suppose, elle aussi, qu'une activité préalable ait eu lieu. Ici l'activité concernée est une activité de discussion (clavardage ou forum) qui doit encourager la collaboration et la participation active des étudiants (p. ex., un débat). Une théorie polyphonique du discours (Trausan-Matu et Rebedea, 2010) fonde la conception de PolyCAFe : les multiples conversations en ligne sont autant de voix jouées par les participants au même moment, et ce widget les analyse pour procurer des rétroactions aux étudiants. Le système a besoin, en entrée, des concepts les plus importants caractérisant le sujet de la discussion, concepts qui peuvent être sélectionnés à partir d'un conceptogramme créé par CONSPECT, ou bien directement spécifiés par l'enseignant ou le tuteur. Ce widget utilise plusieurs techniques de traitement automatique de la langue afin de déterminer les liens implicites entre les tours de parole d'une conversation; ces liens complètent les 
liens explicites générés par les étudiants dans le logiciel de clavardage ou de forum (p. ex., un fil de discussion du forum et les contributions afférentes). Les liens explicites et implicites sont les éléments les plus importants dans la construction d'un graphe de conversation, car ils servent de base à la suite de l'analyse : la détermination des différents fils de discussion, l'identification de l'importance de chaque tour de parole, le calcul des zones à haut niveau de collaboration et la construction d'un sociogramme des participants à la conversation.

À ces différents calculs est combiné un traitement, avec LSA, de la cohérence de chaque tour de parole et de la complétude et importance du contenu du tour de parole par rapport au corpus d'entraînement. Plusieurs types de rétroactions peuvent être présentés aux participants afin de favoriser une réflexion sur leur rôle et leur participation à la conversation, surtout en comparaison avec leurs pairs. De plus, ces rétroactions sont utiles pour préparer de futures discussions (Rebedea et al., 2010). Pour faciliter leur lecture, ces rétroactions sont présentées selon trois niveaux: la conversation en entier, les contributions d'un participant, et le tour de parole. Un outil de visualisation permet d'évaluer l'implication de chaque participant dans la conversation, ainsi que le degré de discours collaboratif tout au long de la discussion. Chacune de ces visualisations est fournie par un widget indépendant, ce qui permet à chaque étudiant de sélectionner l'information qu'il veut voir affichée, et aussi de placer plusieurs widgets sur le même écran pour avoir une vue détaillée de la conversation.

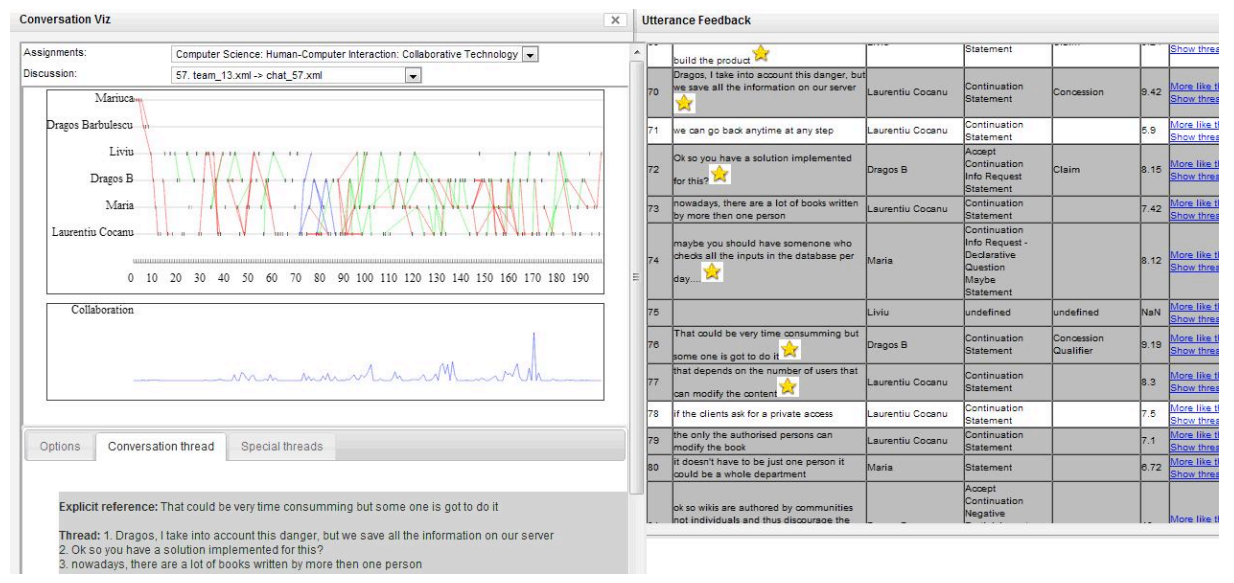

Figure 4. Deux widgets de PolyCAFe : visualisation de la conversation (à gauche) et rétroaction sur les tours de parole (à droite) référant au même tour de parole

Ce widget de visualisation graphique présente des distances principalement sociales, puisqu'il est utilisé pour évaluer le degré de collaboration et d'implication des participants à la conversation. D'autres widgets de PolyCAFe prennent en 
compte des aspects à la fois sociaux et cognitifs de la conversation. Un feedback «participant » présente, pour chaque participant, leur degré de couverture des plus importants concepts de la discussion, mais aussi des indicateurs sociaux comme leur centralité et leur centration moyenne vers les autres participants. Un feedback « tour de parole » surligne les actes de parole de chaque élément de la conversation, et détermine la contribution de chaque tour de parole à l'évolution du fil de discussion. Le feedback «conversation» surligne les concepts les plus importants de la conversation ainsi qu'une liste des concepts reliés, mais qui n'ont pas encore été discutés et pourraient l'être dans une discussion ultérieure. Le feedback "collaboration" signale le nombre moyen de liens par tour de parole, le pourcentage d'argumentations, d'opinions ou de questions, etc.

\section{Un scénario d'utilisation d'un Environnement personnel d'apprentissage et quelques résultats de sa mise en œuvre}

\section{Un scénario d'utilisation}

Avant de décrire plus avant l'environnement personnel d'apprentissage composé des widgets précédents, voici un exemple de scénario-type d'utilisation possible des widgets dans un EPA. Nous présentons une étudiante en informatique, Nora, qui désire approfondir ses connaissances à propos d'AJAX, une méthode de programmation combinant plusieurs technologies web. Elle est inscrite à l'université de Paris et est assistante d'éducation pour payer ses études. Elle dispose de très peu de temps et essaie de l'optimiser. Par exemple, elle déteste attendre plusieurs jours la réponse d'enseignants à ses questions - quand elle les reçoit. Elle a donc décidé d'utiliser un nouveau dispositif d'enseignement à distance qui lui permet à la fois de configurer son environnement de travail selon ses propres habitudes de travail, mais aussi de disposer de rétroactions personnalisées sur ce dernier.

Elle accède à un moteur de recherche spécialisé, iFLSS (en haut à gauche de la figure 5), qui lui permet d'accéder à différentes ressources de diverses communautés web 2.0 (comme del.icio.us, SlideShare, etc.) qui se fondent sur le partage de documents et leur indexation collective, et d'en prendre connaissance. Elle tape "Ajax" dans le champ du moteur et se voit proposer, pour chaque site, un ensemble de documents qu'elle commence à lire. À tout moment, elle peut utiliser à nouveau iFLSS pour avoir des compléments d'information sur telle ou telle notion. Quand elle estime avoir suffisamment de connaissances sur une notion, elle utilise Pensum (en haut à droite de la figure 5) pour en réaliser une synthèse. Cette synthèse, à son tour, peut alimenter CONSPECT qui affiche les concepts traités dans cette dernière et les compare, soit avec une mouture précédente, soit avec la synthèse d'un pair ou d'un expert. Nora peut à tout moment modifier son flux de travail pour solliciter l'un des précédents widgets. De plus, le scénario peut se poursuivre de la façon suivante : Nora peut lire divers documents de cours sur un contenu peu ou pas présent dans sa synthèse, et engager un clavardage avec ses pairs sur ce sujet. PolyCAFe peut être 
utilisé pour analyser la discussion et évaluer sa participation et sa distance avec les autres apprenants et au matériel de cours.

Il est à noter que, si cet article et ce scénario se focalisent sur l'enseignement à distance, l'utilisation de cet EPA peut également se faire dans l'enseignement hybride ou même en présence; les différentes rétroactions n'étant pas censées remplacer celles de l'enseignant, ce dernier peut tirer profit d'un premier niveau d'aide par l'EPA pour procurer aux apprenants une aide ou des conseils plus ciblés, et par là enrichir les interactions enseignant-apprenants.

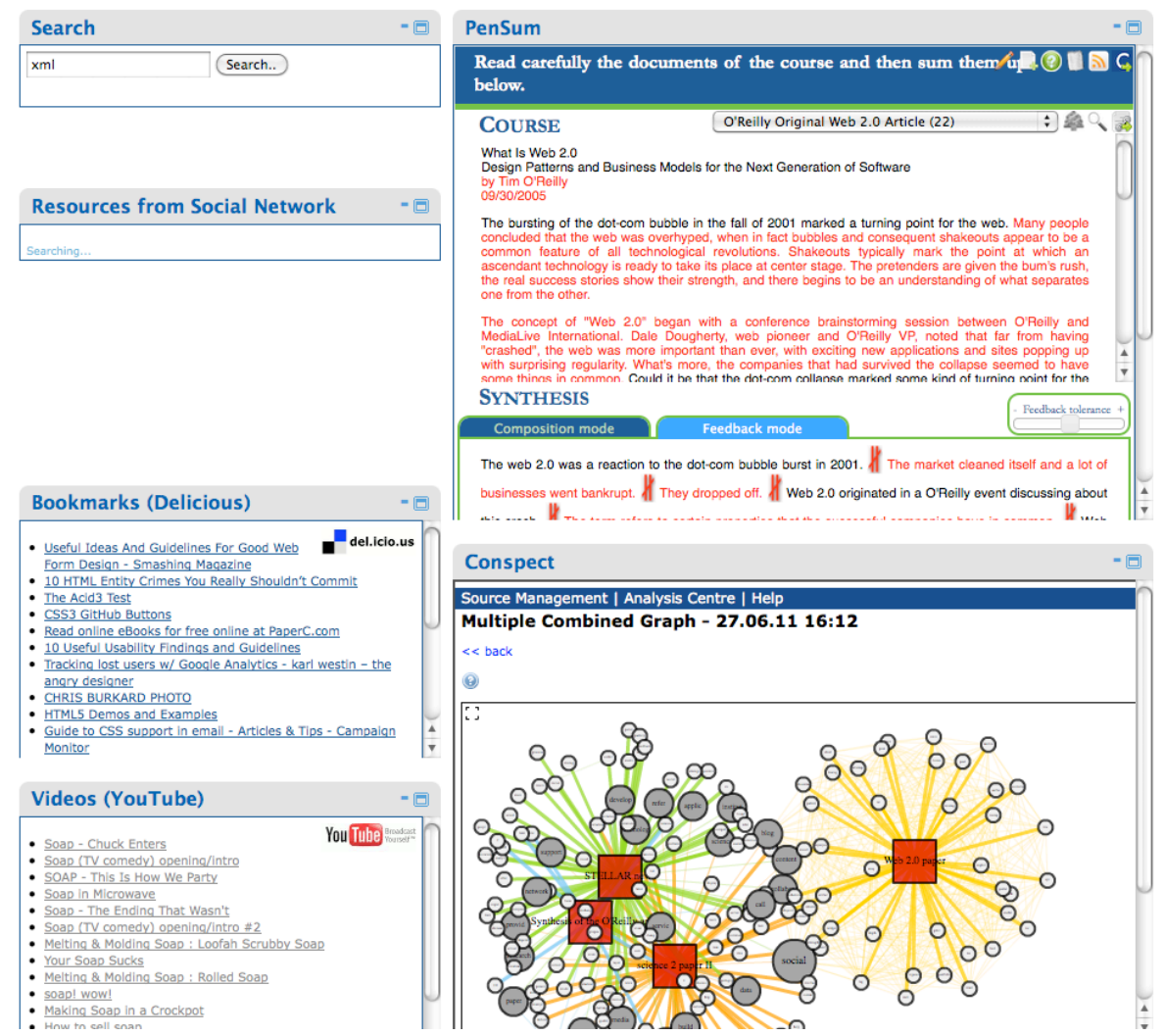

Figure 5. Une copie d'écran de l'environnement personnel d'apprentissage (Wild et al., 2011, p. 40), représentant les widgets iFLSS, Pensum et CONSPECT

Une étude liminaire d'usage a été réalisée à l'université de Bucarest avec 25 participants étudiants en informatique en $4^{\mathrm{e}}$ année d'école d'ingénieurs (Armitt $e t$ al., 2011 ; Wild et al., 2011). Cette étude n'a pas pu, pour des raisons d'organisation, se dérouler à distance, mais les conditions de travail des participants ont été les plus proches possible de telles conditions. Les participants ont réalisé, 
pendant une séance de travail d'une journée, différentes tâches de recherche d'information, de lecture de cours, et de synthèse de ce qu'ils avaient appris en utilisant l'EPA configuré de manière semblable à celui de la figure 5 (PolyCAFe apparaît séparément du fait du grand nombre d'éléments de son interface, mais aussi parce que la tâche à réaliser dans $P o l y C A F e$ peut se réaliser indépendamment de celle des autres widgets).

Le scénario de travail était le suivant et simulait une situation de travail en projet. Les participants étaient répartis en équipes de 3 ou 4 étudiants, formant 8 équipes. Dans une première phase, chaque groupe s'est focalisé dans l'utilisation d'un seul widget (iFLSS, Pensum, CONSPECT, et PolyCAFe) afin d'en comprendre le fonctionnement. Dans une deuxième phase, les étudiants avaient pour tâche de s'engager dans le scénario-type précédemment décrit (celui de Nora), mais avec un contenu différent : celui des standards et technologies web.

Ensuite, ils ont rempli un questionnaire d'acceptation de la technologie issu de celui de Venkatesh et al. (2003) (Unified Theory of Acceptance and Use of Technology, UTAUT), et adapté à la problématique de l'usage d'un EPA. Les items du questionnaire ont été regroupés en 5 catégories (voir tableau 2), complétés par des questions sur des variables telles que le genre, l'âge, le plus haut diplôme, le niveau d'habiletés en informatique et internet des participants. Ils avaient également à répondre aux questions ouvertes suivantes :

- forces et faiblesses de chaque widget individuel utilisé dans la première phase ;

- forces et faiblesses de l'EPA résultant et de la situation de travail ;

- suggestion de nouveaux scénarios d'apprentissage où la combinaison d'au moins deux widgets pourrait être profitable.

Il est à noter que la portée de cette étude est limitée (les étudiants n'ont notamment pas été impliqués dans le test des nouveaux scénarios et des améliorations), et qu'elle a seulement pour but de donner des indications sur la manière dont ces outils sont perçus et utilisés dans un contexte d'enseignement-apprentissage.

Les résultats du questionnaire sont reportés dans le tableau 3 (repris de Wild et al., 2011 , p. 42). Les participants pensent que les bénéfices attendus de l'usage de l'EPA en termes de performance sont modérés $(3,23)$, leur attente d'efforts est assez haute, bien que dispersée, ce qui montre qu'ils pensent que l'EPA ne sera que modérément aisé à maîtriser pour remplir leurs buts d'apprentissage. L'influence sociale est également modérée $(3,08)$, ce qui montre qu'ils sentent qu'ils ne vont pas être spécialement influencés par d'autres (pairs, enseignants) à utiliser 1'EPA. Les conditions facilitatrices sont évaluées comme assez élevées, ce qui montre que les participants pensent avoir les ressources nécessaires et les connaissances pour utiliser l'EPA, même si des points sont encore à améliorer. Enfin, il est à noter que leur intention d'utiliser l'EPA dans l'année à venir est assez basse. Une analyse exploratoire des réponses aux questions ouvertes a été réalisée selon trois 
dimensions: le scénario d'apprentissage proposé, l'interface, la perception des distances. Les résultats sont synthétisés dans le tableau 4.

\begin{tabular}{ll}
\hline Attente de performances & Je trouve que le système serait utile dans mon travail. \\
& L'utilisation de l'EPA me permet de réaliser des tâches plus \\
& rapidement. \\
& L'EPA augmente ma productivité. \\
Attente d'efforts & L'interaction avec l'EPA devrait être claire et compréhensible. \\
& Ce serait facile de devenir compétent dans l'utilisation de \\
& l'EPA. \\
Influence sociale & Les personnes qui sont signifiantes pour moi pensent que je \\
& devrais utiliser l'EPA. \\
& Les personnes qui gèrent mon institution m'ont aidé à utiliser \\
& l'EPA. \\
Conditions facilitatrices & J'ai les ressources nécessaires pour utiliser l'EPA. \\
& J'ai les connaissances nécessaires pour utiliser l'EPA. \\
& Une personne précise (ou un groupe) est disponible pour \\
m'aider quand j'ai des difficultés à utiliser l'EPA. & J'ai l'intention d'utiliser l'EPA dans les 12 prochains mois. \\
Intentions d'usage & me pense que je pourrais utiliser l'EPA dans les 12 prochains \\
& mois. prévois d'utiliser l'EPA dans les 12 prochains mois. \\
\hline
\end{tabular}

Tableau 2. Quelques items du questionnaire UTAUT (Venkatesh et al., 2003, adapté par nos soins)

\begin{tabular}{lccccc}
\hline Catégorie & $N$ & $\min$ & $\max$ & moyenne & écart type \\
\hline Attentes de performance & 25 & 2,00 & 5,00 & 3,23 & 0,75 \\
Attentes d'efforts & 25 & 1,25 & 5,00 & 3,56 & 0,94 \\
Influence sociale & 21 & 1,50 & 4,00 & 3,08 & 0,68 \\
Conditions facilitatrices & 23 & 2,50 & 4,75 & 3,55 & 0,55 \\
Intention d'usage & 24 & 1,00 & 4,67 & 2,79 & 0,99 \\
\hline
\end{tabular}

Tableau 3. Statistiques descriptives des résultats du questionnaire.

Cette analyse permet de mettre en mots les résultats quantitatifs précédemment obtenus à l'aide du questionnaire. Ainsi les widgets utilisés ont été reconnus par les participants comme étant pertinents et simples d'usage dans la réalisation de tâches d'apprentissage. Néanmoins il semblerait que le scénario mis en œuvre ne correspondait pas suffisamment aux besoins des participants, notamment par sa complexité et son absence de réalisme. Les entretiens montrent cependant que les 
étudiants imaginent des possibilités de boucles d'apprentissage faisant intervenir différents widgets. Il est donc possible d'envisager que le scénario ait influencé la perception des participants sur leur usage de l'EPA quant à leurs attentes de performance ; un scénario plus proche de leurs usages quotidiens pourrait donc leur permettre de percevoir davantage l'utilité de ce type de plateforme et faire émerger de nouveaux usages et besoins aussi bien dans les fonctionnalités des outils, que dans les possibilités offertes pour les faire interagir entre eux. De manière générale, les participants mettent en évidence l'importance d'interagir avec l'EPA et de recevoir différents types de rétroactions automatiques sur leur production. Cette étude s'est avérée suffisante pour inférer des améliorations à apporter au niveau de l'affichage des différentes informations notamment pour faciliter l'accès à celles délivrées par les widgets. Toutefois, ce travail appelle à une étude plus extensive, qui permettra d'évaluer l'adéquation des outils avec les apprentissages et leur intégration dans les pratiques des apprenants. Ce type d'information s'avérera nécessaire pour les évolutions ultérieures des widgets, qui devront les prendre en compte avant de reproduire un cycle analogue de conception impliquant les utilisateurs.

\begin{tabular}{|c|c|c|}
\hline Catégorie & Forces & Faiblesses \\
\hline Scénario & $\begin{array}{l}\text { - Chaque widget, individuellement, a } \\
\text { été considéré comme pertinent pour } \\
\text { l'apprentissage } \\
\text { - Des combinaisons de } 2 \text { widgets ont } \\
\text { été suggérées et jugées pertinentes } \\
\text { pour de nombreuses situations } \\
\text { d'apprentissage }\end{array}$ & $\begin{array}{l}\text { - Le scénario mis en œuvre } \\
\text { était trop complexe et peu } \\
\text { réaliste }\end{array}$ \\
\hline $\begin{array}{l}\text { Interface (effet de } \\
\text { la combinaison de } \\
\text { widgets) }\end{array}$ & $\begin{array}{l}\text { - Il est utile d'avoir différents } \\
\text { feedback dans une seule interface } \\
\text { (EPA) } \\
\text { - Il est simple de passer d'une tâche à } \\
\text { l'autre en passant d'un widget à } \\
\text { l'autre }\end{array}$ & $\begin{array}{l}\text { - Des problèmes } \\
\text { d'utilisabilité dus au } \\
\text { voisinage des widgets dans } \\
\text { l'EPA } \\
\text { - Manque d'un standard } \\
\text { visuel entre les différents } \\
\text { widgets }\end{array}$ \\
\hline Distances & $\begin{array}{l}\text { - Bonne combinaison de mesures de } \\
\text { distances entre : résumés des } \\
\text { apprenants et cours, textes écrits } \\
\text { par apprenants et textes d'experts, } \\
\text { apprenants dans la même } \\
\text { discussion }\end{array}$ & $\begin{array}{l}\text { - Des difficultés à apprécier } \\
\text { les nombreuses distances } \\
\text { délivrées par les widgets, } \\
\text { qui sont à des échelles } \\
\text { différentes }\end{array}$ \\
\hline
\end{tabular}

Tableau 4. Synthèse des questions ouvertes à propos de l'usage de l'EPA. 


\section{Discussion}

Nous avons présenté un environnement personnel d'apprentissage composé de trois widgets évaluant des aspects divers des processus d'apprentissage et délivrant leurs évaluations sous la forme de distances (distance sémantique, cognitive individuelle ou sociale). Nous avons montré comment ces trois widgets peuvent s'interconnecter pour former un EPA. Cet environnement a fait l'objet d'une première étude exploratoire d'usage, avec des étudiants en informatique et a ainsi permis d'obtenir de premiers résultats permettant de comprendre comment il a été compris et utilisé, et à plus long terme d'avoir des pistes d'amélioration.

Nous pouvons finalement donner un embryon de réponse à la question qui fédère l'ensemble des articles de ce numéro : «Où va donc la distance? ». Quels types de distances ces nouveaux systèmes mesurent-ils ? Pour favoriser quels types d'activités ? L'ensemble de widgets décrits ici, capables d'échanger des informations sur des écarts épistémiques, qui placent les apprenants dans différentes boucles de rétroaction nous paraît être une réponse prometteuse à cette question. L'activité d'autorégulation, pour être efficacement mise en œuvre par l'apprenant, nécessite de disposer d'outils de type « instruments» qui indiquent clairement à ce dernier sa position (qu'elle soit épistémique ou sociale). Il convient bien évidemment de rester vigilant afin de ne pas enfermer l'apprenant dans des « you loops » (Pariser, 2011) qui, en sur-personnalisant les résultats des requêtes, ne lui apporteraient pas de matériel suffisamment nouveau et susceptible d'entraîner un apprentissage et «normaliserait» l'activité entière. Nous pensons toutefois que les techniques de traitement automatique des langues, même si elles sont imparfaites, sont maintenant suffisamment avancées pour permettre un premier rang d'interactions pour l'apprenant, afin qu'il porte un regard réflexif sur son travail, avant même que l'enseignant n'entre dans la boucle, ce dernier se focalisant ainsi sur des tâches de plus haut niveau.

Le scénario proposé, même s'il est limité et de ce fait pourrait être considéré comme peu authentique, a eu le mérite de proposer aux apprenants un tour d'horizon des possibilités des widgets. Ceux-ci, malgré leurs imperfections, ont été considérés par les étudiants comme des outils pertinents dans leur apprentissage. Ces résultats sont encourageants puisque les étudiants s'approprient relativement bien les informations destinées à réduire les distances en vue de faciliter leurs tâches d'apprentissage. Les échanges avec les étudiants ont par ailleurs fait émerger, de leur part, des possibilités d'usage conjoints des widgets. Ce type de compréhension des outils est d'autant plus favorable qu'il nous semble être une condition d'appropriation et à plus forte raison quand il s'agit, comme c'est le cas ici, d'outils destinés à être utilisés en autorégulation et combinés en fonction des besoins. Bien évidemment, l'usage d'un tel EPA doit être observé avec des utilisateurs plus novices et dans des disciplines et situations variées et certainement plus écologiques que celles de cette étude exploratoire.

Une étude de plus grande envergure, qui se déroulerait à distance et laisserait les étudiants s'approprier les outils comme bon leur semble, permettrait une évaluation 
plus approfondie. Les outils pourraient chacun faire l'objet d'un apprentissage préalable et être évalués individuellement, et l'effet de leurs combinaisons pourrait être également mesuré, dans une perspective centrée sur les outils ou bien dans l'activité d'autorégulation de l'apprentissage, rarement prise en compte dans l'enseignement à distance (Vovides et al., 2007). Dans de tels contextes, les apprenants réalisent fréquemment de nombreuses activités, que nous avons déjà listées dans l'introduction. Si de nombreux systèmes favorisant ces activités existent dans la littérature, ils ont encore des limites : ils sont indépendants, centrés sur une seule activité ; ils ne procurent pas de retours centrés sur des caractéristiques de haut niveau des textes produits. Et c'est finalement la première limite qui est la plus décisive : si le fait de s'atteler à des tâches plus complexes comporte des difficultés nouvelles, le fait de pouvoir cumuler les points de vue à travers différentes applications aptes à interagir (même avec des éléments de plus bas niveaux) nous semble une piste viable pour l'amélioration des EPA, en attendant d'obtenir plus d'informations sur les manières de favoriser les pratiques d'apprentissage via les outils et leur combinaison.

\section{Remerciements}

Les recherches évoquées dans cet article ont été en partie financées dans le cadre $\mathrm{du}$ projet européen LTfLL (Language Technologies for Lifelong Learning), $7^{\mathrm{e}}$ PCRD, 2008-2011. Les auteurs remercient les deux relecteurs anonymes pour leurs judicieux conseils.

\section{Bibliographie}

Armitt G., Stoyanov S., Hensgens J., Smithies A., Braidman I., Mauerhofer C., et al., Deliverable 7.4 LTfLL Validation 4, Heerlen, OUNL, 2011.

Attwell G., "Personal Learning Environments - the future of eLearning?", eLearning Papers, vol. $2, n^{\circ} 1,2007$, p. 1-8.

Berlanga A. J., van Rosmalen P., Boshuizen H. P. A., Sloep P. B., "Exploring formative feedback on textual assignments with the help of automatically created visual representations", Journal of Computer Assisted Learning, à paraître, doi: 10.1111/j.13652729.2011.00425.x.

Butler D. L., Winne P. H., "Feedback and self-regulated learning: A theoretical synthesis", Review of Educational Research, vol. 65, n 3, 1995, p. 245-281.

Carver C. S., Scheier M. F., "Self-regulation of action and affect", K. D. Vohs et R. F. Baumeister (Eds.), Handbook of self-regulation. Research, theory, and applications ( $2^{\text {nd }}$ ed.), Guilford, New York, 2011, p. 3-21.

Dessus P., "Loops, gaps and contradiction: Three PLE objects that foster learning", MUPPLE Lectures (Knowledge Media Institute of the Open University), Milton Keynes (UK), 2011. 
Dessus P., Trausan-Matu S., van Rosmalen P., Wild F. (Eds.), Proc. Natural Language Processing in support of Learning: Metrics, feedback and connectivity, Brighton, AIED, 2009.

Dessus P., «Qu'est-ce que l'enseignement ? Quelques conditions nécessaires et suffisantes de cette activité », Revue Française de Pédagogie, vol. 164, 2008, p. 139-158.

Dessus P., « Les effets de la distance sur le contenu d'un cours : une analyse avec LSA », Revue de l'Éducation à Distance, vol. 18, $\mathrm{n}^{\circ} 2,2003$, p. 61-73.

Hattie J., Timperley H., “The power of feedback”, Review of Educational Research, vol. 77, $\mathrm{n}^{\circ} 1,2007$, p. 81-112.

Landauer T. K., Dumais S. T., “A solution to Plato's problem: the Latent Semantic Analysis theory of acquisition, induction and representation of knowledge", Psychological Review, vol. $104, \mathrm{n}^{\circ} 2,1997$, p. 211-240.

Loiseau M., Dupré D., Dessus P., « Pensum, un système d'aide à la compréhension de cours à distance », M. Bétrancourt, C. Depover, V. Luengo, B. D. Lièvre et G. Temperman (Eds.), Conférence EIAH 2011, Presses de l'université de Mons, Mons, 2011, p. 287-299.

Moore M. G., "Theory of transactional distance", D. Keegan (Ed.), Theoretical Principles of Distance Education, Routledge, New York, 1993, p. 22-38.

Olivier B., Liber O., Lifelong Learning: The need for portable Personal Learning Environments and supporting interoperability standards, Bristol, The JISC Centre for Educational Technology Interoperability Standards, Bolton Institute, Rapport de recherche, 2001.

Pariser E., The filter bubble: What the internet is hiding from you, New York, Penguin, 2011.

Rebedea T., Dascalu M., Trausan-Matu S., Banica D., Gartner A., Chiru C., et al., "Overview and preliminary results of using PolyCAFe for collaboration analysis and feedback generation", M. Wolpers, P. Kirschner, M. Scheffel, S. Lindstaedt \& V. Dimitrova (Eds.), Sustaining TEL: From Innovation to Learning and Practice (EC-TEL 2010) (vol. 6383), Springer Berlin, 2010, p. 420-425.

Saba F., Shearer R. L., "Verifying key theoretical concepts in a dynamic model of distance education”, The American Journal of Distance Education, vol. 8, n 1, 1994, p. 36-59.

Stahl, G., Group cognition. Computer support for building collaborative knowledge, Cambridge, MIT Press, 2006.

Trausan-Matu S., Dessus P. (Eds.), Proc. Natural Language Processing in support of Learning: Metrics, feedback and connectivity, Bucharest, Matrix Rom, 2010.

Trausan-Matu S., Rebedea T., "A polyphonic model and system for inter-animation analysis in chat conversations with multiple participants", A. Gelbukh (Ed.), Computational Linguistics and Intelligent Text Processing (CICLing 2010), Springer, New York, 2010, p. 354-363.

Trausan-Matu S., Stahl G., Sarmiento J., "Supporting polyphonic collaborative learning", Eservice Journal, vol. 6, $\mathrm{n}^{\circ} 1,2007$, p. 58-74. 
Trope Y., Liberman N., "Construal-level theory of psychological distance", Psychological Review, vol. 117, n², 2010, p. 440-463.

Venkatesh V., Morris M. G., Davis G. B., Davis F. D., "User acceptance of information technology: toward a unified view”, MIS Quarterly, vol. 27, n 3425-478, 2003.

Vovides Y., Sanchez-Alonso S., Mitropoulou V., Nickmans G., “The use of e-learning course management systems to support learning strategies and to improve self-regulated learning", Educational Research Review, vol. 2, n 1, 2007, p. 64-74.

Westerhout E., Monachesi P., Markus T., Posea V., "Enhancing the learning process: Qualitative validation of an informal learning support system consisting of a knowledge discovery and a social learning component", M. Wolpers, P. A. Kirschner, M. Scheffel, S. Lindstaedt et V. Dimitrova (Eds.), Sustaining TEL: From innovation to learning and practice (EC-TEL 2010), Springer, Berlin, 2010, p. 374-389.

Wild F., Ullmann T., Scott P., Rebedea T., Hoisl B., "Applicability of the Technology Acceptance Model for widget-based Personal Learning Environments", Proceedings of the $1^{\text {st }}$ Workshop on Exploring Fitness and Evolvability of Personal Learning Environments, La Clusaz, 2011. 